1 How prepared are pharmacists to support atrial fibrillation patients in adhering to newly

2 prescribed oral anticoagulants?

3

4

5

6

7

8

\title{
Introduction
}

Atrial fibrillation (AF) is the most common cardiac arrhythmia in clinical practice with an estimate of 1.36 million people in England living with the condition. ${ }^{1}$ It is a major predisposing factor for stroke, increasing the risk of individuals by fivefold and contributing to approximately $20 \%$ of all ischaemic strokes. ${ }^{2-4}$ Oral anticoagulants (OACs) have been shown to be the most effective agents for the prevention of AF-related strokes and there is extensive evidence demonstrating their underuse in practice. ${ }^{5-7}$ Vitamin $\mathrm{K}$ antagonists (VKAs), predominantly warfarin, have been the main OAC used in England for prevention of AF-related strokes. ${ }^{7}$ The availability of Non-vitamin K antagonist Oral Anticoagulants (NOACs) have shown to be non-inferior to warfarin and the latest National Institute of Health and Care Excellence (NICE) guidelines for AF recommend the choice of using a VKA or a NOAC based on patients' clinical features and preferences with a personalised package of care. ${ }^{8}$ This intention is to encourage patients' involvement in shared decision making as well as recognising the opportunity to minimise the reported wastage of medicines due to nonadherence. $^{9}$

Patients starting on VKA in England traditionally attend a dedicated anticoagulation management service for monitoring their treatment and are more closely supported by a healthcare professional upon initiation. ${ }^{8,10}$ Option of VKA patient self-monitoring is also available and taken up by a small number of patients. ${ }^{11,12}$ Those that are started on a NOAC do not require regular blood monitoring and guidelines recommend a first follow up appointment four weeks following treatment initiation. ${ }^{13}$ Although these features can raise a potential concern on adherence, it is unclear whether VKAs or NOACs are a better option for 
improved adherence, since most recent studies focus on comparison of adherence patterns within NOACs. ${ }^{14,15}$

Challenges of patient adherence in the context of oral anticoagulants used for stroke prevention in AF are similar to those that arise from other long term conditions (LTC), with evidence illustrating a third to half of patients are likely to stop taking their medication, resulting in a negative impact on their quality of life, mortality and the NHS economy ${ }^{16-18}$. Studies have also demonstrated patients are more likely to become non-adherent in the first month of being initiated a new medicine for $\operatorname{LTCs}^{19}$. Patients newly initiated on medicines frequently encountered unmet needs for information and required additional support. Hence, an intervention, based on a perceptions and practicalities approach ${ }^{20,21}$, recognising adherence is influenced by beliefs and/ or symptoms that are unique to each patient was proposed, where pharmacists adopt a patient centred approach by listening to the patient, providing information and reassurance in response to expressed needs during telephone consultations. ${ }^{22}$ This intervention was shown to improve adherence and led to the development of a nationally commissioned service called New Medicine Service (NMS). This service was implemented into practice in 2011 as part of the Advanced Services under the National Health Service (NHS) community pharmacy contractual framework, whereby community pharmacist can opt to provide the service from their pharmacy. ${ }^{23}$

In 2014 an evaluation of the NMS was undertaken and showed to improve adherence by $10 \%$ to people who were started on new medication for asthma/COPD, type 2 diabetes, and hypertension or antiplatelet/anticoagulant treatment. The NMS comprises three consultations arranged between the pharmacist and patient over a month following initiation of any new medicine in the above mentioned indications. Each visit allows the pharmacist to determine 
51 personal barriers that may contribute to non-adherence and most importantly, facilitate appropriate interventions with individual patients' that are tailored to support their needs ${ }^{24}$. To undertake this role, community pharmacists acquire a number of skills and knowledge that can address the clinical and behavioural components required for supporting adherence. At present pharmacists have access to a range of learning materials developed to support the introduction

of the NMS and are required to sign a declaration of competency. However, an assessment of pharmacists' needs in providing a NMS consultation on oral anticoagulants has not been formally undertaken.

59

\section{Aim of the study}

To assess community pharmacists' current practice, perceived skills and knowledge in supporting patients' medication adherence as part of NMS on OAC for stroke prevention in patients with AF. Also, we intended to evaluate pharmacists' perceived needs for additional support or education to increase the implementation of the service.

Ethical approval

Ethical approval was not sought because this survey was an assessment involving no changes to the current services being delivered. The questionnaire was anonymous and pharmacists were free to decline participation. As such, the principles of ethical research, such as confidentiality and anonymity, were followed.

Method

A cross-sectional survey was developed a) to benchmark community pharmacists' existing practice and experience of providing a NMS on OAC for stroke prevention in patients with 
emphasis on supporting adherence and c) to identify training needs and pharmacists' preference of resources for a NMS consultation. Data were collected using an anonymous online survey that was distributed to pharmacists working within community pharmacies in London by email with a link through their Local Pharmaceutical Committees (LPCs). The survey was also advertised by the Royal Pharmaceutical Society (RPS) through their local practice forum to encourage participation.

A literature search identified a lack in published surveys assessing community pharmacist knowledge, skills and NMS provision on OAC for stroke prevention. Hence, questions were developed internally by author consensus and pre-testing to a sample of twenty community pharmacists and five hospital pharmacists. Feedback was used to ensure the questions were meaningful and to examine their clarity and acceptability. Pilot results were not included in the final analysis, but used to modify and adapt the questions and format (Appendix A). Data obtained from the application of the final version was used to validate the survey (as detailed in statistical analysis), ensuring credible results.

\section{$\underline{\text { Survey }}$}

The survey was designed using Survey Monkey and comprised a summary description of the study and questions designed to collate demographics and professional characteristics of pharmacists, including gender, years of experience and educational background. The current practice of community pharmacist was determined by quantifying the NMS consultation(s) undertaken by respondents in a three month period for all commissioned LTC and more specifically oral anticoagulants. The survey was then organised in several sections, where statements were provided for participants to rate: 
a) Pharmacists' knowledge and confidence based on their perception, subdivided into when advising patients on NOACs and on VKA(5-point Likert scale ranging from strongly agree to strongly disagree). their London pharmacy stores.

b) Perceived helpfulness of information sources available (4-point Likert scale ranging from not helpful to very helpful)

c) Attributed importance to a list of counselling information to be discussed with patients newly prescribed an OAC (4-point Likert scale ranging from critical to not important).

d) Resources used during a NMS consultation for oral anticoagulants (list provided and answered as yes/no), followed by an open-ended question for those with a 'yes' response to enter the resource description.

e) Pharmacists' preference of support material required when discussing oral anticoagulant with patients (4-point Likert scale ranging from critical to not important), with an option of documenting additional resources and/ or tools that were not highlighted.

f) Pharmacists' views on the importance of soft skills for improving their NMS service provision for OAC (4-point Likert, ranging from very helpful to not helpful).

All questions were designed with a skip pattern and respondents had the opportunity to free type additional information for each question.

The first e-mail invitation was sent on the 4th December 2014 and the survey remained open until $31^{\text {st }}$ January 2015. Reminder e-mails were sent on three different occasions to community pharmacists through LPCs and large multiple community pharmacies' regional managers were also contacted directly to confirm receipt of online survey and encourage its dissemination to 
126 The number of pharmacists working in community pharmacies within London was 3807, 127 representing $28 \%$ of the workforce in community pharmacy ${ }^{22}$. Assuming that $10 \%$ of these 128 could be delivering the NMS for $\mathrm{OAC}^{25}$, using a confidence interval of $95 \%$ and accepting a $1293.5 \%$ error we expected to receive 277 answers.

$\underline{\text { Data analysis }}$

132

The data were analysed in SPSS version 23. Validation of the survey comprised confirmatory factor analysis and reliability analysis (Cronbach's alpha). Data analysis comprised descriptive univariate statistics and bivariate analysis (Wilcoxon test). A confidence level of $95 \%$ was considered.

\section{Results}

A total of 269 responses were received over a two month period, of which 12 were excluded as they only contained demographic data. The majority were male $(62 \% ; n=159)$, with a wide range of experience in practice. Most responders were permanent members of staff. Approximately a third had undertaken further academic qualifications following their registration (table 1).

\section{Please insert table 1 here}

The survey's validation indicated it comprised 5 domains, all of them with high validity, ranging from 0.676 to 0.892 . Detailed information of the survey's validity is presented in table 2. 
In a three month period, $87 \%$ of pharmacists completed one or more NMS consultations, $68 \%$ completed one or more NMS for OAC, and 35\% completed one or more NMS for NOACs.

Data indicates that $25 \%$ of pharmacists had completed six or more NMS consultations for all OAC, of which $11 \%$ were for NOACs (figure 1).Those with extra academic qualifications undertook more NMS for OAC $(\mathrm{p}=0.012)$, whilst proprietor pharmacists undertook less $(\mathrm{p}=0.035)$.

Please insert figure 1 here

Pharmacists were more confident in their knowledge, skills and access to resources for VKA than for NOAC $(\mathrm{p}<0.005)$. Pharmacists with extra qualifications expressed higher confidence on all the dimensions illustrated in figure 2, except for using a counselling check list, which they use as little as all pharmacists $(\mathrm{p}<0.05)$. The results also highlight pharmacists' unfamiliarity with NOAC alert cards, as a significantly lower proportion checked for them in comparison with VKA alert cards $(\mathrm{p}<0.001)$, albeit there is also room for improvement in the knowledge on NOAC, comparable to $23 \%$ for VKA (figure 2).

Please insert figure 2 here

All counselling items were deemed important, although with varying importance (figure 3).

The key priorities for pharmacist during their consultation with patient newly initiated on oral anticoagulant was firstly to discuss what actions to take when bleeding occurs, followed by supporting adherence. In this order, the basic mode of action was perceived as the least important dimension when discussing newly initiated OAC with AF patients for stroke prevention. 
175 The importance of a patient's alert card was perceived as critical to very important by $78 \%$ of 176 pharmacists, more often by those that stated to routinely check for an alert card. Both the 177 correlation coefficients for VKA and NOACs were statistically significant, although weak $178(\mathrm{r}=0.304 ; \mathrm{p}<0.001$ for VKA and $\mathrm{r}=0.187 ; \mathrm{p}=0.005)$.

Please insert figure 3 here

Around half the pharmacists confirmed they utilised one source of information during a NMS consultation on OAC (51\%). The most frequently mentioned was the British National Formulary (BNF), the sole source utilised by a quarter of pharmacists. Online resources were accessed by $34 \%$, of which a third did not specify the websites used. The most popular medical online websites named included patient websites, NHS choice, NICE clinical knowledge summaries and electronic medicine compendium to reach Patient Information Leaflets and/or Summaries of Product Characteristics.

The most valued resources to have available during a NMS consultation on OAC in AF patients for stroke prevention were check-lists, followed by patient information leaflets, with insignificant differences (figure 4).

Please insert figure 4 here

196 Soft skills were acknowledged as important to attain to address the behavioural dilemmas of adherence by over $80 \%$ of pharmacists. We have considered these as less tangible constructs, not clinical and often related to social and administrative sciences, such as communication 
skills $^{25}$. Within the latter, counselling skills were deemed the most necessary for an effective NMS consultation, albeit all skills were considered important (figure 5).

\section{Please insert figure 5 here}

The preferred training delivery methods for additional education were online educational modules $(49.1 \%$ considered as very helpful), followed by clinical case studies $(43.3 \%)$ and practical workshops $(43.2 \%)$. The least favoured delivery methods within the list were independent study and clinical supervision, with only $24.1 \%$ and $16.7 \%$ respectively of pharmacist viewing these as very helpful. Seminars or lectures and facilitated group discussions were seen as very helpful by $30.5 \%$ and $30.0 \%$, respectively.

\section{Discussion}

Over eighty five percent of community pharmacists in London are undertaking NMS consultations, of which OAC make up a small proportion. The DoH commissioned report for evaluating the NMS has shown antiplatelet and anticoagulants contributed to $8.5 \%$ of all NMS consultations, in line with our findings ${ }^{26}$. NHS England latest national pharmaceutical list 2013 2014 reported a quarter of community pharmacies in London do not provide $\mathrm{NMS}^{27}$. This value is above our results, finding only $13 \%$ of pharmacists did not provide this nationally commissioned service. This suggests inconsistencies in access to the NMS services by patients in London and potentially inequalities in the care and support of adherence for their LTC medication, undermining the core principles of NHS. All AF patients initiated on oral anticoagulant for stroke prevention within London should be provided with an equal opportunity to access the NMS support systems rather than occur by chance ${ }^{28}$. 
Proprietor pharmacists were undertaking fewer NMS consultations, as indicated by the DoH report $^{27}$. This finding requires further exploration to determine if independent pharmacies require support in adopting the NMS as part of routine practice, especially as the number of independent contractors in London was estimated to be $61 \%$ of total community pharmacies in $2013-14^{27}$.

Only for NOACs there was no positive relationship between higher education levels and the number of NMS consultations undertaken, which may largely be explained by the minimal exposure community pharmacists have to the patients initially prescribed a NOAC in London. Most current local arrangements across London for initiation of NOAC are largely dependent on shared care guidelines that require secondary care to continue supplying the first two to three months of NOAC and for most patients the NMS is not embedded as part of their care pathway. Furthermore the uptake of NOAC prescribing in London during our survey data collection period was averaged at $6 \%$ of the total number of OAC prescriptions, resulting in less opportunity for community pharmacist to undertake a NMS consultation. Further work is required to embed the NMS on oral anticoagulant for stroke prevention in the care pathway of patients with $\mathrm{AF}$ as routine practice.

The level of knowledge and confidence of the pharmacist responding to this survey demonstrates a wide variation, with approximately twice as many feeling uncertain or lack the confidence in their knowledge on NOAC, in comparison with VKA. The unfamiliarity and minimal exposure to NOAC is reflected in their knowledge and confidence. Conversely, VKA have been available for many years with national standardised recommendations and resources for patients and healthcare professionals including community pharmacists ${ }^{15}$. These include emphasis on pharmacists' role with the importance of ensuring patients on an OAC receive 
appropriate verbal and written information at the start and throughout their treatment including a patient safety alert card. The availability of NOAC alert cards is currently dependent on the individual manufacturers, possibly contributing to the low proportion reporting to check for a NOAC alert card and highlights the need to make pharmacists aware and adopt a single oral anticoagulant alert card.

Responding pharmacists' primary focus during a consultation with a patient was safety followed by adherence to OAC. This reflects the responding pharmacists' own perceptions of their responsibilities that lay in the traditional risk averse roles and is leading to the importance of patients being able to recognise and manage bleeding as a priority to supporting adherence during their consultation. Awareness of our findings is pivotal and should be incorporated into training programmes for NMS on oral anticoagulant for prevention of AF-related strokes.

Facilitating a NMS consultation requires skills beyond clinical knowledge that create the correct conditions and behaviours to support patient adherence and lead to safe and effective compassionate care. Almost all pharmacist acknowledged the importance of utilising the appropriate soft skills to build trust and a professional relationship from the start of the consultation. Counselling and coaching skills were also deemed important by the majority for creating the correct conditions and behaviours to support patients' adherence.

There was an expressed need for a concise resource to use in practice as a reference guide or access to relevant information when undertaking a NMS consultation. The predominant resource used was BNF, containing legal and professional guidelines, focusing on the clinical effectiveness and safety of medicines but lacking information related to the process or factors impacting on adherence. The diversity of online and other resources accessed may potentially lead to variation in the quality of information used during the consultation. The majority of pharmacist felt the availability of a check-list and patient information leaflets (PIL) during their 
274 NMS consultation would be of great benefit. At present there is no single online platform that

275 is available for pharmacist to access information and resources to support their consultation on

276 OAC in England. The American Anticoagulation forum is an example of such a platform that

277 could be adapted to the UK pharmacists' needs by incorporating relevant validated tools,

278 videos, PIL and alert cards ${ }^{29}$.

279 Online modules were rated as the most helpful education method. The remaining methods highly rated correspond to the existing options used to deliver continuous professional development to pharmacist. Clinical supervision was one of the least favoured, probably because it is not part of routine community practice.

\section{Strengths and Limitations}

Although the response rate $(6.8 \%)$ was low, the sample reached was in line with the estimate.

Responses originated from all geographical areas of London with a variety in their demographic characterisation, making the results here presented valuable information. Nonetheless, it should be acknowledged that the sample largely represents experienced pharmacists, limiting extrapolations.

\section{Conclusion}

This survey suggests that community pharmacists' current practice in London is limited in undertaking NMS consultations for OAC, particularly for NOACs for the prevention of AFrelated strokes. Pharmacists perceive their knowledge and skills as having some gaps, implying there is an urgent need to revisit the currently implemented training programme for community pharmacists and to develop the most appropriate resources to support consultations, leading to wider dissemination of the service. 
Acknowledgements: The authors wish to acknowledge all the pharmacists who tool their time to answer the survey.

Funding: This study did not receive any funding. All costs were supported by the researchers and the organisations involved.

Conflicts of interests: Both Mr. Antoniou and Dr. Costa have received travel grants/honararia from Bayer to present at conferences during the current year. Mr. Antoniou has also received honararia from Daiichi-Sankyo, Boehringer, Pfizer/BMS. None of these, however, directly relate to the work being presented here. The remaining authors declare that they have no financial relationships that might lead to a conflict of interest.

\section{References}

1. Public Health England. Atrial Fibrillation estimates in England: Application of recent population estimates of AF in Sweden. 2015. https://www.gov.uk/government/uploads/system/uploads/attachment_data/file/612288 /AtrialfibrillationprevalenceestimatesinEngland.pdf. Accessed 22 June 2017.

2. Wolf, P.A., Abbott, R.D., Kannel, W.B. Atrial fibrillation as an independent risk factor for stroke: the Framingham Study. Stroke 1991; 22: 983-8

3. Marini, C., De Santis, F., Sacco, S., Russo, T., Olivieri, L., Totaro, R. \& Carolei, A. Contribution of atrial fibrillation to incidence and outcomes of ischaemic stroke: results from a population-based study. Stroke 2005;36:1115-19

4. Royal College of Physicians Sentinel Stroke National Audit Programme (SSNAP). Clinical audit April-June 2014 report prepared by Royal College of Physicians, 
Clinical Effectiveness and Evaluation Unit on behalf of the Intercollegiate Stroke Working Party

5. Atrial fibrillation investigators. Risk factors for stroke and efficacy of antithrombotic 
12. Anticoagulation Self-monitoring Alliance. Anticoagulation services and patient access to INR self-monitoring in the NHS in England. 2014. http://www.anticoagulationeurope.org/files/files/ACSMA\%20Anticoagulation\%20Ser vices\%20in\%20England\%20Report\%20.pdf. Accessed 24 June 2017.

13. Heidbuchel, H., Verhamme, P., Aligns, M., Antz, M., Hacke, W., Oldgren, J. et al. European Heart Rhythm Association practical guide on the use of new oral anticoagulants in patients with non-valvular atrial fibrillation. Europace 2013; 15: 625651.

14. Lichten, C., Castle-Clarke, S., Manville, C., Horvath, V., Robin, E., Krapels, J. et al. The future of anticoagulation management in atrial fibrillation in Europe. An assessment of today's challenges with recommendation for the future. RAND Europe 2015.

15. McHorney, C., Crivera, C., Laliberté, F., Nelson, W.W., Germain, G., Bookhart, B., et al. Adherence to Non-VKA Oral Anticoagulant Medications Based on the Pharmacy Quality Alliance Measure. Current Medical Research and Opinion 2015. doi: $10.1185 / 03007995.2015 .1096242$

16. National Institute for Health and Care Excellence. Medicine Optimisation: the safe and effective use of medicines to enable the best possible outcome (NG5). March 2015. https://www.nice.org.uk/guidance/ng5/resources/medicines-optimisation-the-safeand-effective-use-of-medicines-to-enable-the-best-possible-outcomes-51041805253. Accessed 15 January 2015

17. World Health Organisation. Adherence to long term therapies. Evidence for action. 2003 
18. Haynes, R.B., Ackloo, E., Sahota, N., McDonald, H.P. \& Yao, X. Interventions for enhancing medication adherence (Review). Cochrane Database of Systematic Reviews. 2008;2(2).

19. Barber, N., Parsons, J., Clifford, S, Darracott, R. \& Horne, R. Patients problems with new medication for chronic conditions. Qual Saf Health care 2004;13:172-175.

20. Horne, R. Compliance, adherence and concordance. In: Taylor K, Harding G, eds. Pharm Pract. London: Taylor and Francis, 2001:165-84

21. Horne, R. R, Weinman, J., Barber, N., Elliot, R., Morgan, M., Cribb, A. \& Kellar, I. Concordance, Adherence and Compliance in Medicine Taking: A conceptual map and research priorities. London: NCCSDO, 2005,40-6

22. Clifford, S., Barber, N., Elliott, R., Hartley, E. \& Horne, R. Patient-centred advice is effective in improving adherence to medicines. Pharmacy World and Science 2006; 28(3): 165-170.

23. Pharmaceutical Service Negotiating Committee. New Medicine Service [Internet]. 2011.

24. Elliott, R., Boyd, M., Waring, J., Barber, N., Mehta, R., Chuter, A., \& Avery, A. Understanding and appraising the new medicines service in the NHS in England. (2014) A randomised controlled trial and economic evaluation with qualitative appraisal comparing the effectiveness and cost effectiveness of the New Medicines Service in community pharmacies in England. Nottingham: The School of Pharmacy University of Nottingham.

25. Boyle, C.J. and Robinson, E.T. Leadership is Not a Soft Skill. American Journal of Pharmaceutical Education 2013; 77 (10) Article 209

26. Health Education England. The community pharmacy workforce in London 2015: Executive summary 
27. Health \& Social Care Information Centre. General Pharmaceutical Services. England $\begin{array}{llll}2004-05 & \text { to } & \text { 2013-14. November }\end{array}$ http://www.hscic.gov.uk/catalogue/PUB15933/gen-pharm-eng-201314-Report.pdf. Accessed 14 January 2015.

28. National Health Service. The NHS constitution. The NHS belongs to us all. 2013. https://www.gov.uk/government/publications/the-nhs-constitution-for-england. Assessed 24 June 2017.

29. Anticoagulation forum. http://www.acforum.org. Accessed 14 January 2015 